\title{
Profile and treatment outcomes of patients with tuberculosis in Northeastern Ethiopia: a cross sectional study.
}

\author{
Daniel Mekonnen ${ }^{1,2}$, Awoke Derbie $^{1}$, Hailu Mekonnen ${ }^{3}$, Yohannes Zenebe ${ }^{1}$
}

\author{
1. Department of Medical Microbiology Immunology and Parasitology, College of Medicine \\ and Health Sciences, Bahir Dar University, Bahir Dar, Ethiopia \\ 2. Biotechnology Research Institute, Bahir Dar University, Bahir Dar, Ethiopia \\ 3. Departments of Curative Treatment, Raya Kobo Woreda Health Office, Kobo, Ethiopia
}

\begin{abstract}
:
Background: Data on the epidemiology of tuberculosis and its treatment outcomes were incomplete in the study area and this study was done to fill this gap.

Methods: Institution based cross sectional study was conducted from January 2011 to December 2014. A total of 949 TB patients who were on treatment in North Eastern Ethiopia, Eastern Amhara region were included. Data was analyzed using SPSS version 20. Frequency, percentages and means were used to present data. To assess the associations of treatment outcomes with sex, age, type of TB and human immunodeficiency virus (HIV), logistic regression was used.

Results: The proportion of smear positive and negative pulmonary TB, and extra pulmonary TB were 187/949 (19.7\%), $322 / 949$ (33.9\%) and 440/949 (46.4\%), respectively. Treatment success rate was 853/949 (89.9\%). Smear positive pulmonary TB and TB/HIV co-infections were significantly associated with unsuccessful treatment outcome, $\mathrm{P} \leq 0.002$.

Conclusion: Extra pulmonary TB was the most prevalent types of TB followed by smear negative pulmonary TB. Treatment success rate was above the WHO target of $85 \%$. The causes for the high proportion of smear negative PTB and EPTB should be further investigated. Special emphasis should be put on smear positive PTB patients and TB/HIV co-infected patients to decrease unsuccessful treatment outcome and TB transmissions.

Keywords: Tuberculosis, types of tuberculosis, treatment outcomes, Ethiopia.

DOI: http://dx.doi.org/10.4314/ahs.v16i3.4

Cite as: Mekonnen D, Derbie A, Mekonnen H, Zenebe Y. Profile and treatment outcomes of patients with tuberculosis in Northeastern Ethiopia: a cross sectional study. Afri Health Sci 2016;16(3): 663-670. DOI: http:/ / dx.doi.org/10.4314/ abs.v16i3.4
\end{abstract}

\section{Background}

Tuberculosis (TB) is a disease of centuries. It killed more persons than any other infectious diseases ${ }^{1}$. Tuberculosis is a disease of poverty and mainly affects poor countries. A person with TB loses, on average, $20-30 \%$ of annual household income due to illness².

Acid fast bacilli (AFB) microscopy with spot-morningspot sputum sample collection strategy was the recom-

\section{Corresponding author: \\ Daniel Mekonnen, \\ Department of Medical Microbiology \\ Immunology and Parasitology, \\ College of Medicine and Health Sciences, \\ Bahir Dar University, Bahir Dar, Ethiopia \\ +251912990288 P.O.Box:1383 \\ Email: nigusdaniel@gmail.com}

mended technique for the diagnosis and follow-up of pulmonary TB (PTB) patients in the Directly Observed Treatment short course (DOTS) program ${ }^{3-5}$. Effective treatment of TB started in 1946, with the introduction of streptomycin (SM). The main problem with current chemotherapy is too long duration of treatment regimen thus allowing opportunities for interruptions in drug taking that may lead to the emergence of drug resistance ${ }^{6}$.

Globally, the rate of treatment success among new smearpositive PTB who were treated in the 2012 cohort was $86 \%$. Treatment success rate was higher among smear positive PTB than smear negative PTB $^{7}$. In Ethiopia in the same year, TB treatment success rate among new TB cases was $91 \%{ }^{5}$. However; it is unknown among retreated cases $^{5}$.

There was no data about TB treatment outcome in the study area in particular. In addition, aggregate national

African Health Sciences Vol 16 Issue 3, September 2016 
data might not reflect the real TB profile and treatment outcomes due to subtle socioeconomic, geographic and methodological variations. Thus, studies like this might show the appropriate proportion of mean treatment outcome and types of TB in the specific area. Thus, this study was done to determine the profile and treatment outcome of TB patients in Amhara National Regional State (ANRS); Kobo and Raya Kobo Woredas of Ethiopia.

\section{Materials and methods \\ Study setting, period and population}

Institution based cross-sectional study was conducted on patients diagnosed and treated over the period of January 2011 to December 2014 in Kobo, Robit and Gobiye health centers. These health centers found in eastern Amhara region in north wollo zone, $552 \mathrm{~km}$ away from Addis Ababa. The woredas are part of a mid-altitude area which lies between the Ethiopian Highlands to the west and the Afar depression to the east. Currently, there are 8 additional health centers in the studied area that provide TB diagnosis and treatment. However, these health centers were newly opened and had incomplete registration system to be included in the study.

\section{Tuberculosis diagnosis and treatment strategy}

The pulmonary TB diagnosis and treatment was based on the stop TB strategy. That is, sputum samples were collected using spot-morning-spot strategy and three slides were prepared, one from each sample. Slides were processed and examined using the standard Ziehl-Neelsen (ZN) AFB staining technique . $^{8}$

Extra pulmonary TB was diagnosed at nearby government and private health institutions primarily based on clinical data with limited service of imaging and pathological examinations. Diagnosis of TB in children was based on clinical and X-ray findings.

For those patients diagnosed with active TB, the standard TB treatment regimen; 2(RHZE)/4 (RH) and $2 \mathrm{~S}$ (HRZE)/1(HRZE)/5(HR) E were administered for new and previously treated TB patients respectively ${ }^{9}$.

\section{Operational definitions}

Health Center: A primary health care unit that serves a population of 15,000-25,000 and is comprised of specialized clinics like TB, antiretroviral treatment (ART), maternal and child health clinics.

\section{Inclusion and exclusion criteria}

All TB patients with complete demographic and clinical data were included in the study. Whereas, patients with unknown types of TB and treatment outcomes, including transferred out were excluded.

\section{Data collection and tools}

Demographic and clinical data such as age, sex, type of TB, TB treatment outcome, status of human immunodeficiency virus (HIV) infection, co-trimoxazole prophylaxis (CPT) and antiretroviral treatment (ART) status were retrieved from TB registry using data extraction sheets.

\section{Data analysis}

Collected data was entered, cleared, and analyzed using SPSS statistical software package (IBM Corp. Released 2011. IBM SPSS Statistics for Windows, Version 20.0. Armonk, NY: IBM Corp.). Frequency, percentage, mean and standard deviation (SD) were used to present data. To assess the associations of treatment outcome with sex, age, type of TB and HIV status; stepwise logistic regression model was used. Variables with a p-value of $\leq 0.2$ PubMed in bi variate analysis were included in the multivariate analysis.Associations were determined using odds ratio and 95\% confidence interval. A p-value of < 0.05 was considered to indicate statistical significance.

\section{Ethics statements}

This study was approved by Amhara Regional Health Bureau Institutional Ethical Review Board (IRB) to utilize the data. No patient details that may link to the patient identity were used and the confidentially was maintained.

\section{Results}

\section{Demographic epidemiology of tuberculosis}

Among the total of $990 \mathrm{~TB}$ patients registered on TB notification books, $30(3 \%)$ were transferred out and 11 $(1.1 \%)$ were with unknown treatment outcomes. We excluded these categories of patients. Thus, data from a total of 949 TB patients were included for analysis. Of the total included, 528/949 (55.4\%) were males. Mean age of participants were 32.8 years with SD of 16.3. Moreover, high proportions of TB patients; 285/949 (30\%) and $212 / 949(22.3 \%)$ were in the age groups of $25-34$ and 15-24 years, respectively [Table 1]. 
Table 1: Distribution of demographic characteristics and HIV status among tuberculosis patients in Kobo and Raya Kobo Woredas-Ethiopia, 2015.

\begin{tabular}{|c|c|c|c|c|c|}
\hline \multirow[b]{2}{*}{ Variables } & \multicolumn{5}{|c|}{ Type of Tuberculosis } \\
\hline & & $\begin{array}{l}\mathrm{PTB}^{+} \\
\mathrm{N}(\%)\end{array}$ & $\begin{array}{c}\text { PTB }^{-} \\
\text {N }(\%)\end{array}$ & $\begin{array}{l}\text { ЕРТВ } \\
\mathrm{N}(\%)\end{array}$ & $\begin{array}{c}\text { Total } \\
\text { N }(\%)\end{array}$ \\
\hline \multirow[t]{2}{*}{ Sex } & Female & $91(48.6)$ & $133(41.3)$ & $197(44.7)$ & $421(44.5)$ \\
\hline & Male & $96(51.4)$ & $189(58.7)$ & $243(55.3)$ & $528(55.5)$ \\
\hline \multirow[t]{7}{*}{ Age } & $<=14$ & $5(2.7)$ & $29(9)$ & $61(13.9)$ & $95(10)$ \\
\hline & $15-24$ & $51(27.3)$ & $66(20.5)$ & $95(21.6)$ & $212(22.3)$ \\
\hline & $25-34$ & $70(37.4)$ & $94(29.2)$ & $121(27.5)$ & $285(30)$ \\
\hline & $35-44$ & $25(13.4)$ & $43(13.3)$ & $53(12)$ & $121(12.7)$ \\
\hline & $45-54$ & $18(9.6)$ & $44(13.6)$ & $47(10.7)$ & $109(11.5)$ \\
\hline & $55-64$ & $11(5.9)$ & $21(6.5)$ & $38(8.6)$ & $70(7.4)$ \\
\hline & $>=65$ & $7(3.7)$ & $25(7.7)$ & $25(5.7)$ & $57(6)$ \\
\hline \multirow[t]{3}{*}{ HIV Status } & Unknown & $6(3.2)$ & $6(1.8)$ & $6(1.4)$ & $18(1.9)$ \\
\hline & Positive & $65(34.8)$ & $87(27)$ & $75(17)$ & $227(24)$ \\
\hline & Negative & $116(62)$ & $229(71.1)$ & $359(81.6)$ & $704(74.2)$ \\
\hline Total & & $187(19.7)$ & 322 (33.9) & $440(46.4)$ & 949 (100) \\
\hline
\end{tabular}

\section{Profile of tuberculosis}

The proportion of smear positive PTB, smear negative PTB and EPTB were 187/949 (19.7\%), 322/949 (33.9\%) and 440/949 (46.4\%), respectively [Table 1]. There was no significant difference on the types of TB with regard to sex and age of participants. The proportion of TB/ HIV co-infection was 227/949 (24\%). Moreover, TB/ HIV co-infection was twice higher among smear positive PTB than EPTB [Table 1].

\section{Tuberculosis treatment outcomes}

The overall unsuccessful treatment outcome was 96/949 $(10.1 \%)$ wherein failed, died and lost to follow up shared at $21 / 949(2.2 \%), 47 / 949(5 \%)$ and $28 / 949$ (2.9\%) respectively. It is found that the proportion of unsuccessful TB treatment outcome was higher among male and older age patients than their counterparts with no statistical significance. On the other hand, the proportion of cured was at 132/949 (13.9\%) and treatment completed at $721 / 949(76 \%)$ [Table 2]. 
Table 2: Distribution of tuberculosis treatment outcomes by demographic and profile of TB patients, Kobo and Raya Kobo Woredas-Ethiopia, 2015.

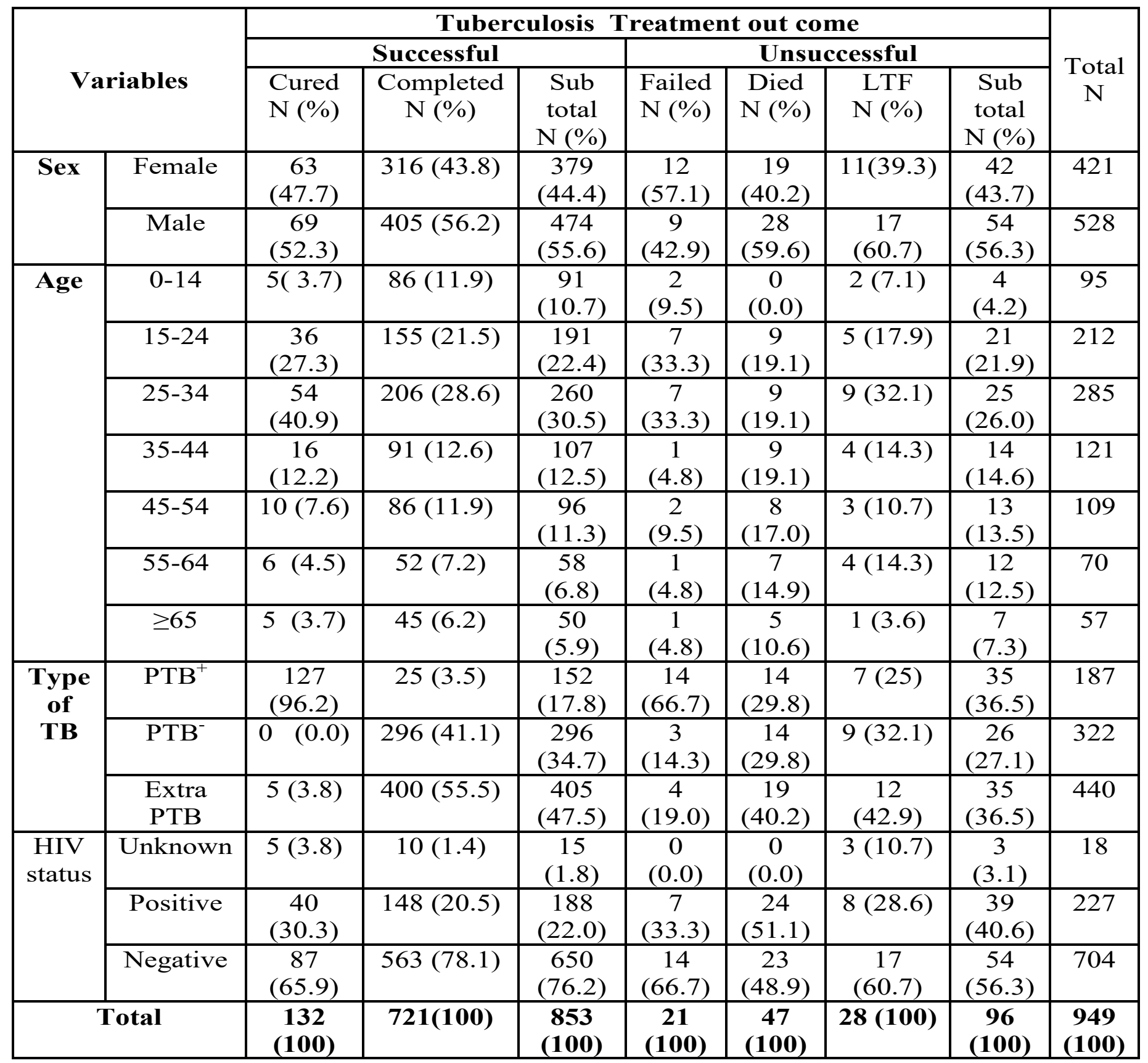

Furthermore, significant differences were observed on treatment outcome between smear positive PTB and
EPTB, and between HIV positive and HIV negative TB patients [Table 3]. 
Table 3: Association of unsuccessful treatment outcomes with possible predictors among tuberculosis patients, Kobo and Raya Kobo Woreda-Ethiopia, 2015.

\begin{tabular}{|c|c|c|c|c|c|}
\hline \multirow[t]{2}{*}{ Variables } & \multicolumn{3}{|c|}{ TB treatment outcome } & \multirow{2}{*}{$\begin{array}{l}\text { Over all P-value } \\
\text { of binary } \\
\text { regression }\end{array}$} & \multirow{2}{*}{$\begin{array}{l}\text { AOR (95\% CI), } \\
\text { P-value }\end{array}$} \\
\hline & $\begin{array}{l}\text { Unsuccessful } \\
\mathrm{N}(\%)\end{array}$ & $\begin{array}{l}\text { Success full } \\
\mathrm{N}(\%)\end{array}$ & $\begin{array}{l}\text { Total } \\
\text { (N) }\end{array}$ & & \\
\hline \multicolumn{4}{|l|}{ Sex: } & 0.89 & \\
\hline Male & $54(56.3)$ & $474(55.6)$ & 528 & & \multirow[t]{2}{*}{-} \\
\hline Female & $42(43.7)$ & $379(44.4)$ & 421 & & \\
\hline \multicolumn{4}{|l|}{ Age: } & 0.17 & \\
\hline $0-14$ & $4(4.2)$ & $91(10.7)$ & 95 & & $0.3(0.08-1), 0.06$ \\
\hline $15-24$ & $21(21.8)$ & $191(22.4)$ & 212 & & $0.64(0.2-1.6), 0.35$ \\
\hline $25-34$ & $25(26.0)$ & $260(30.5)$ & 285 & & $0.4(0.16-1.07), 0.07$ \\
\hline $35-44$ & $14(14.6)$ & $107(12.5)$ & 121 & & $0.65(0.24-1.7), 0.4$ \\
\hline $45-54$ & $13(13.5)$ & $96(11.3)$ & 109 & & $0.79(0.3-2.1), 0.66$ \\
\hline $55-64$ & $12(12.5)$ & $58(6.8)$ & 70 & & $1.3(0.5-3.8), 0.5$ \\
\hline$\geq 65$ & $7(7.3)$ & $50(5.9)$ & 57 & & 1 \\
\hline \multicolumn{4}{|c|}{ Types of TB: } & 0.0001 & \\
\hline PTB+ & $35(36.5)$ & $152(17.8)$ & 187 & & $2.3(1.3-3.8), 0.02$ \\
\hline PTB- & $26(27.1)$ & $296(34.7)$ & 322 & & $0.88(0.5-1.5), 0.6$ \\
\hline EPTB & $35(36.4)$ & $405(47.5)$ & 440 & & 1 \\
\hline \multicolumn{4}{|c|}{ HIV Status: } & $\mathbf{0 . 0 0 0 1}$ & \\
\hline Unknown & $3(3.1)$ & $15(1.8)$ & 18 & & $2.6(0.7-9.6), 0.15$ \\
\hline Positive & $39(40.6)$ & $188(22.0)$ & 227 & & $2.6(1.6-4.27), 0.001$ \\
\hline Negative & $54(56.3)$ & $650(76.2)$ & 704 & & 1 \\
\hline Total & $96(10.1)$ & 853 (89.9) & 949 & & \\
\hline
\end{tabular}

\section{Discussion}

This study revealed high proportion of TB among males than females. This agreed with different findings reported in Ethiopia and aboard ${ }^{5,10-13}$. The possible reason might be related with, lower rates of notification of TB among women than men due to low health seeking behavior, lack of financial control and stigma associated with being TB positive 2 . Majority, at $22.3 \%$ and $30 \%$ of TB patients were in the age groups of 15-24 and 25-34 years respectively. This report is also in line with other literatures $5,10,14-15$ which indicated that, TB mainly affects the reproductive segment of the population. This might be due to high HIV prevalence in these age groups (personal opinion).

The TB profile data showed that high proportions of TB patients were EPTB and smear negative PTB which is comparable with other Ethiopian TB profile data ${ }^{16}$. However, it is in contrary with reports from outside Ethiopia 5,14,17-18. Currently, two justifications seems plausible: the role of false positive ${ }^{20}$ over prescription/ and the zoonotic transmission, social and host-pathogen genomic factors $^{21}$. These two justifications are presented as follows;

African Health Sciences Vol 16 Issue 3, September 2016 
The first argument discussed that, most of EPTB and smear negative PTB cases were diagnosed and transferred in from private health facilities. However, these facilities might not have well organized facilities and expertise to diagnose EPTB and smear negative PTB cases. This is in line with studies by Iwnetu et al. and Ehlers et al . According to these studies, up to $15 \%$ of all EPTB and 97.3\% smear negative PTB cases were diagnosed wrong$1 y^{19-20}$. Furthermore, clinicians might abused the low sensitivity of $\mathrm{ZN}$ and low specificity of pathological tests and might rushed to diagnose presumptive TB cases as confirmed smear negative PTB/EPTB (expert opinion)

The other justification goes in line with Berg et al, study justified that regular and direct contact with live animals, was a significant risk factor for EPTB ${ }^{21}$. These factors also seem likely, because, people in the study area are known live stock keepers and due to their local tradition, most of their domestic animals shared human shelter. Moreover, these people regularly use raw meat and milk. Moreover, the high smear negative PTB might be due to difference in the immunological and nutritional status of Ethiopian with other counties (unpublished report). However, to identify the real cause of such high prevalence report in Ethiopia, it needs further comparative ecological and cohort study.

In the present study, the proportion of TB/HIV co-infection was at 227/949 (24\%), much higher than WHO report of the year 201522. Moreover, TB/HIV co-infection was twice higher among smears positive PTB than EPTB which is in contrary with a literature ${ }^{23}$. This can be explained by tailoring to Ethiopian situation. If the reports of Iwunetu et al. and Ehlers et al., mentioned above, considered as a possible cause for high rate of EPTB and smear negative PTB in Ethiopia, this would also dilute the TB/HIV co-infection rate among EPTB and smear negative PTB patients.

The odd of having unsuccessful treatment outcome was 2.3 times higher among smear positive PTB than EPTB patients (AOR: 2.3; 95\% CI: 1.3-3.8; P: 0.02). This might be due to higher HIV prevalence, $(34.8 \%)$ among smear positive PTB than smear negative PTB (27\%) and EPTB $(17 \%)$ [Table 1]. Similar finding was reported from Addis $\mathrm{Ababa}^{24}$.
The odds of having unsuccessful TB treatment outcome was 2.6 times higher among TB/HIV co-infected than HIV negative TB patients (AOR: 2.6; 95\% CI: 1.6-4.27; $\mathrm{p}<0.0001$ ) (Table 3). This might be due to low level of immunity and drug mal-absorption ${ }^{25}$. This finding was in line with a study from Addis Ababa, Eastern Ethiopia, Benin and Malawi ${ }^{15,24,26,-27}$. Furthermore, the proportion of unsuccessful treatment outcome was higher among TB/HIV co-infected people with no history of CPT and ART than those on CPT and ART [data not shown]. Administration of ART is recommended during TB therapy regardless of the CD4 cell count, ${ }^{28}$ because ART is associated with reduction in mortality and HIV disease progression ${ }^{29-32}$. Moreover, all HIV-infected patients with active TB should receive $\mathrm{CPT}^{30,22}$

\section{Limitation of the study}

The recorded data lack detail clinical profile like CD4 count and other comorbidies. On top of this, due to the inherent limitation of the study design, the study couldn't show any cause effect relation between significantly associated variables.

\section{Conclusions and recommendations}

The proportion of smear negative PTB and EPTB were higher than smear positive PTB cases. Plus, the overall TB treatment success rate was above the WHO target of $>85 \%$. Moreover, smear positive PTB and TB/HIV coinfections were significantly associated with unsuccessful TB treatment outcomes. Thus, we would like to forward the following recommendations; (1) the causes for the high proportion of smear negative PTB and EPTB cases should be further investigated. (2) The ART and CPT coverage must reach $100 \%$ to TB/HIV co-infected patients so that their unsuccessful treatment outcome rate would be improved. (3) Especially emphasis should be paid to smear positive PTB patients to decrease unsuccessful treatment outcome and decrease transmissions.

\section{Acknowledgements}

Authors express deep appreciation to Amhara National Regional Health Bureau Research and technology department for approving the proposal. Moreover, we also thank North Wollo Zonal Health Department, Kobo and Raya Kobo Woreda Health Offices. 


\section{Funding}

This research received no specific grant from any funding agency

\section{Competing interests:}

We the authors declare that we have no competing interests.

\section{Authors' contributions}

DM substantially contributed in the design, statistical analysis, interpretation of data and drafting the manuscript. AD was involved in the data collection and entry, statistical analysis and enriched the manuscript. HM was involved in data collection, entry, and analysis. YZ critically revised the table and whole manuscript for intellectual contents. All the authors read and approved the final manuscript.

\section{References}

1. Palomino C, Leao C, Ritacco V.Tuberculosis.From basic science to patient care. Institute of Tropical Medicine (Belgium, Brazil, Argentina). www.Tuberculosis Textbook.com.2007.

2. World Health Organization. Gender and Tuberculosis. Department of Gender and Women's Health 20, Avenue Appia. Geneva, Switzerland. WHO 2002.

3. Keeler E, Perkins D, Small P et al. Reducing the global burden of tuberculosis: the contribution of improved diagnostics. Nature 2006.

4. Federal Democratic Republic of Ethiopia Ministry of Health. Comprehensive Training Manual for Clinical and Programmatic Management of TB, Leprosy and TB/ HIV. Participants' Manual. Addis Ababa. 2012.

5. World Health Organization. Global tuberculosis report. WHO/HTM/TB/2014.08. Geneva, Switzerland. WHO 2014.

6. Mitchison D and Davies G. The chemotherapy of tuberculosis: past, present and future. Int J Tuberc Lung Dis 2012; 16(6): 724-732.

7. World Health Organization. Global tuberculosis report. WHO/HTM/TB/2013.11. Geneva, Switzerland. WHO 2013.

8. Lawn D, Zumla I. Tuberculosis. Lancet 2011; 378: 5772.

9. Federal Democratic Republic of Ethiopia. Ministry of Health. Guideline for Clinical and Programmatic Man- agement of TB, Leprosy and TB/HIV in Ethiopia. Addis Ababa, Ethiopia 2012. www.etharc.org/resources/download/finish/33/709. Accessed 13 May 2015.

10. Tessema B, Muche A, Bekele A et al. Treatment outcome of tuberculosis patients at Gondar University Teaching Hospital, Northwest Ethiopia. A five year retrospective study. BMC Public Health 2009, 9:371.

11. Ditah C, Reacher M, Palmer C et al. Monitoring tuberculosis treatment outcome: analysis of national surveillance data from a clinical perspective. Thorax 2008.

12. Berg S, Schelling E, Hailu E et al. Investigation of the high rates of extrapulmonary tuberculosis in Ethiopia reveals no single driving factor and minimal evidence for zoonotic transmission of Mycobacterium bovis infection. BMC Infect Dis. 2015; 15:112.

13. World Health Organization. Global tuberculosis report. WHO/HTM/TB/2012.6. Geneva, Switzerland. WHO 2012.

14. Endris M, Moges F, Belyhun Y et al. Treatment Outcome of Tuberculosis Patients at Enfraz Health Center, Northwest Ethiopia: A Five-Year Retrospective Study. Hindawi Publishing Corporation, Tuberculosis Res and Treatment 2014; 2014: 7 PubMed pages.

15. Ade S, Harries AD, Tre 'bucq A et al. National Profile and Treatment Outcomes of Patients with Extrapulmonary Tuberculosis in Be'nin. PLoS ONE 2014; 9(4).

16. World Health Organization. Globaltuberculosis report.Geneva, Switzerland. WHO 2012.

17. Gebretsadik B, Enquselassie F and Aseffa A. Treatment outcome of smear-positive pulmonary tuberculosis patients in Tigray Region, Northern Ethiopia. BMC Public Health 2012; 12:537.

18. Sisay S, Mengistu B, Erku W et al. Directly Observed Treatment Short-course for tuberculosis control program in Gambella Regional State, Ethiopia: ten years experience. BMC Res Notes 2014; 7:44.

19. Iwnetu R, van den Hombergh J, Woldeamanuel $Y$ et al. Is tuberculous lymphadenitis over-diagnosed in Ethiopia? Comparative performance of diagnostic tests for mycobacterial lymphadenitis in a high-burden country. Scand J Infect Dis 2009;41(6-7):462-8. PubMed

20. Ehlers VJ, Aragaw GS. An audit of diagnosis and treatment of tuberculosis in Ethiopia. Afr J Prm Health Care Fam Med 2014; 6 (1): 6 pages.

21. Berg S, Schelling E, Hailu E et al. Investigation of the high rates of extrapulmonary tuberculosis in Ethio- 
pia reveals no single driving factor and minimal evidence for zoonotic transmission of Mycobacterium bovis infection. BMC Infect Dis. 2015; 15:112.

22. Onyebujoh PC, Ribeiro I, Whalen CC et al. Treatment Options for HIV-Associated Tuberculosis. I Infect Dis. 2007 August 15; 196 (1): 35-45.

23. American Thoracic Society, Centers for Disease Control and Prevention, Infectious Diseases Society of America. Treatment of tuberculosis. Am J Respir Crit Care Med. 2003;167: 603-2. reprinted in MMWR Recomm Rep 2003; 52 (11):1-77.

24. Hailu D, Abegaz W.E and Belay M: Childhood tuberculosis and its treatment outcomes in Addis Ababa: a 5-years retrospective study. BMC Pediatrics 2014; 14:61. 25. Luetkemeyer A. Tuberculosis and HIV. HIV InSite Knowledge Base Chapter. Comprehensive, up-to-date information on HIV/AIDS treatment, prevention, and policy from the University of California San Francisco. January 2013. available at [http://hivinsite.ucsf.edu/ InSite?page $=\mathrm{kb}-05-01-06]$.

26. Amante T.D, Ahemed T.A. Risk factors for unsuccessful tuberculosis treatment outcome (failure, default and death) in public health institutions, Eastern Ethiopia. Pan Afr Med J. 2015; 20:247.

27. Tweya H, Feldacker C, Phiri S, Ben-Smith A, Fenner
L, Jahn A, Kalulu M, Weigel R, Kamba Ch, Banda R, Egger M, Keiser O. Comparison of Treatment Outcomes of New Smear-Positive Pulmonary Tuberculosis Patients by HIV and Antiretroviral Status in a TB/HIV Clinic, Malawi. PLoS ONE 2013; 8(2).

28. World Health Organization. Rapid Advice: Antiretroviral Therapy for HIV Infection in Adults and Adolescents. 2009.

29. Girardi E, Palmieri F, Angeletti C, Vanacore P, Matteelli A, Gori Andrea, Carbonara S, and Ippolito G: Impact of Previous ART and of ART Initiation on Outcome of HIV-Associated Tuberculosis. Hindawi Publishing Corporation. Clinical and Developmental Immunology. 2012; 2012: 8 pages.

30. World Health Organization. Guidelines on Co-Trimoxazole Prophylaxis for HIV-Related Infections among Children, Adolescents and Adults in Resource-Limited Settings: Recommendations for a Public Health Approach. August 2006.

31. Suthar AB, Lawn SD, del Amo J et al. Antiretroviral Therapy for Prevention of Tuberculosis in Adults with HIV: A Systematic Review and Meta Analysis. PLoS Med 2012; 9(7).

32. Chakaya J, Getahun H, Granich R Confronting TB/ HIV in the era of increasing anti-TB drug resistance. J Int AIDS Soc 2008; 11:6. 